Quim. Nova, Vol. 33, No. 1, 70-75, 2010

\title{
EMISSÕES NATURAIS E ANTRÓPICAS DE METAIS E NUTRIENTES PARA A BACIA INFERIOR DO RIO DE CONTAS, BAHIA
}

\author{
Francisco C. F. de Paula \\ Departamento de Ciências Agrárias e Ambientais, Universidade Estadual de Santa Cruz, 45650-000 Ilhéus - BA, Brasil \\ Luiz Drude de Lacerda*, Rozane V. Marins e José E. Aguiar \\ Instituto de Ciências do Mar, Universidade Federal do Ceará, 60165-081 Fortaleza - CE, Brasil \\ Álvaro R. C. Ovalle \\ Centro de Ciências Biológicas e Biotecnologia, Universidade Estadual do Norte Fluminense, 28015-620 Campos dos Goytacazes \\ - RJ, Brasil \\ Cézar A. T. Falcão Filho
}

Universidade Estadual de Santa Cruz, 45650-000 Ilhéus - BA, Brasil

Recebido em 15/12/08; aceito em 21/7/09; publicado na web em 25/11/09

\begin{abstract}
NATURAL AND ANTHROPOGENIC EMISSIONS OF METALS AND NUTRIENTS TO THE LOWER CONTAS RIVER BASIN, BAHIA STATE, BRAZIL. Emission factors of anthropogenic activities and natural processes were used to estimate nutrients and metals loads to the Contas River lower basin, Southern Bahia, Brazil. Among natural sources, emission from soil leaching is larger for $\mathrm{N}, \mathrm{Cu}$ and $\mathrm{Pb}$. Atmospheric deposition is the major natural source of $\mathrm{P}, \mathrm{Zn}, \mathrm{Cd}$ and $\mathrm{Hg}$. Among anthropogenic sources, agriculture is the major source of N, Cu and P. Urban sources are the major contributors to the other elements. Present anthropogenic land uses are already responsible for 78 and $99 \%$ of total $\mathrm{N}$ and $\mathrm{P}$ loads and about $50 \%$ of total $\mathrm{Cu}$ and $\mathrm{Hg}$.
\end{abstract}

Keywords: emission factors; pollutants; Rio de Contas estuary.

\section{INTRODUÇÃO}

Os principais nutrientes com elevado potencial de eutrofização $(\mathrm{N}$ e $\mathrm{P}$ ) e metais de interesse ambiental ( $\mathrm{Zn}, \mathrm{Cu}, \mathrm{Cd}, \mathrm{Pb}$ e o $\mathrm{Hg}$ ) são contaminantes com presença frequente e ubíqua em vários efluentes de atividades humanas incluindo industriais, urbanos e agropecuários. Além disto, são componentes naturais da deposição atmosférica, de solos, águas e biota. Dentre as fontes antrópicas, aquelas de origem urbana apresentam grande potencial de contaminação de águas superficiais por esses elementos oriundos da disposição inadequada de rejeitos sólidos urbanos (lixões), ${ }^{1-3} \mathrm{de}$ águas servidas (esgotamento sanitário) lançadas no meio ambiente sem tratamento prévio adequado ${ }^{4}$ e runoff urbano, resultante da lixiviação por águas da chuva de superfícies urbanizadas. ${ }^{5-7} \mathrm{Em}$ áreas rurais, atividades agrícolas também são importantes fontes de $\mathrm{N}, \mathrm{P}$ e de alguns metais, particularmente $\mathrm{Cd}, \mathrm{Cu}, \mathrm{Pb}, \mathrm{Hg}$ e $\mathrm{Zn}$, presentes como resíduos em fertilizantes, como micronutrientes $(\mathrm{Cu}$ e $\mathrm{Zn})$ e também como princípio ativo de biocidas $(\mathrm{Cu}) \cdot{ }^{8-12}$ Da mesma forma a atividade pecuária também utiliza diversos insumos contendo metais, eventualmente liberados com as fezes e urina dos animais, juntamente com elevadas concentrações de $\mathrm{P}$ e N. ${ }^{13-15}$ Portanto, mesmo em áreas de baixo desenvolvimento industrial e urbano nutrientes e alguns metais podem se constituir em ameaças potenciais à biodiversidade e à qualidade dos recursos hídricos e biológicos da região.

Nutrientes e metais atingem os estuários principalmente associados ao material particulado em suspensão e/ou dissolvidos na coluna d'água dos sistemas fluviais e ainda por deposição atmosférica. As características hidrogeoquímicas das regiões estuarinas e costeiras favorecem a deposição e acumulação dessas substâncias que podem assim atingir concentrações elevadas, mesmo em áreas onde fontes pontuais significativas destes contaminantes estão ausentes. ${ }^{16}$

\footnotetext{
*e-mail: ldrude@pq.cnpq.br
}

No recorte nacional das Regiões Hidrográficas do Brasil, ${ }^{17}$ a Região do Atlântico Leste compreende uma série de bacias hidrográficas de médio porte (entre 10.000 e 100.000 km²), as quais deságuam no oceano entre os paralelos 10 e $20^{\circ} \mathrm{S}$. Esta região pode ser caracterizada por possuir cidades pequenas a médias, baixo índice de industrialização e onde a atividade econômica é centrada na agricultura e pecuária. Outra semelhança que ocorre entre essas bacias é a carência de estudos sistemáticos sobre a biogeoquímica das águas e sedimentos de seus corpos hídricos, estuários e regiões costeiras adjacentes. A bacia hidrográfica do Rio de Contas é um exemplo típico desta região.

Sínteses globais sobre o fluxo de materiais continentais para os oceanos, resultantes de programas internacionais em larga escala, e.g. IGBP-LOICZ (Land-Ocean Interaction in the Coastal Zone), WAVES (Water Availability, Vulnerability of Ecosystems and Society), ELOISE (European Land-Ocean Interactions Studies), têm sistematicamente ressaltado a escassez de dados relativos às bacias de pequeno e médio porte onde praticamente inexistem estudos sistemáticos. Na América do Sul, e mais especificamente no litoral brasileiro, as grandes bacias hidrográficas (>100.000 km²) já estão incluídas nos balanços globais gerados por estes programas, porém não são ainda consideradas as bacias de pequeno e médio porte. ${ }^{18}$

A grande dificuldade da inclusão de bacias hidrográficas de pequeno e médio porte nos cenários globais dos fluxos continentais para os oceanos é a inexistência de medidas sistemáticas in situ de cargas, fluxos e concentrações. Uma alternativa aceitável se constitui no uso de fatores de emissão das substâncias de interesse, estimados a partir de dados de produção, censos populacionais e rurais, processos produtivos e características dos processos naturais e antrópicos que mobilizam as substâncias de interesse no ambiente. Estes parâmetros encontram-se, via de regra, disponíveis para a maioria das bacias hidrográficas brasileiras, o que torna o uso de fatores de emissão uma importante ferramenta para a quantificação dos aportes de elementos e substâncias para bacias hidrográficas, além de fornecerem 
estimativas consistentes para regiões onde não estejam disponíveis dados específicos de concentrações e fluxos. ${ }^{19,20}$

No presente estudo é apresentada uma estimativa das cargas naturais e antrópicas de $\mathrm{N}, \mathrm{P}, \mathrm{Zn}, \mathrm{Cu}, \mathrm{Cd}, \mathrm{Pb}$ e $\mathrm{Hg}$ para a bacia inferior do Rio de Contas, Estado da Bahia, que foi obtida através do uso de fatores de emissão para os diferentes processos naturais e antrópicos que atuam nessa região. Os resultados são comparados com bacias similares da região NE do Brasil onde estudos semelhantes estão disponíveis ${ }^{21,22}$ e validados através da comparação com os dados disponíveis sobre a distribuição destes elementos em diferentes compartimentos ambientais da bacia inferior ${ }^{23-25}$ e resultados de composição elementar de amostras de sedimentos de fundo em áreas selecionadas da bacia inferior.

\section{PARTE EXPERIMENTAL}

\section{Área de estudo}

A bacia do Rio de Contas pertence às bacias do Atlântico Leste e possui uma área total de $55.000 \mathrm{~km}^{2}$. É a maior bacia hidrográfica inteiramente contida no Estado da Bahia, estendendo-se de forma alongada no sentido leste-oeste por aproximadamente $700 \mathrm{~km}$, conectando três biomas: o Cerrado na Chapada Diamantina (região de cabeceiras), a Caatinga em seu trecho médio e a Mata Atlântica na bacia inferior. Sua foz está localizada na cidade de Itacaré (14 18 'S; $\left.39^{\circ} \mathrm{W}\right) .{ }^{23} \mathrm{O}$ Rio de Contas apresenta caráter eminentemente torrencial e, com exceção do canal principal, todos os afluentes dos trechos médio e superior são intermitentes, resultando em deflúvios da ordem de 13 e $17 \mathrm{~m}^{3} \mathrm{~s}^{-1}$, respectivamente. No posto fluviométrico mais a jusante da bacia (Ubaitaba), já na bacia inferior a vazão média anual do deflúvio é de 4 a 5 vezes maior $\left(100 \mathrm{~m}^{3} \mathrm{~s}^{-1}\right) .^{24,25}$

A bacia inferior é definida a partir da última quebra abrupta de seu perfil longitudinal, na Usina Hidroelétrica (UHE) de Funil e possui cerca de $7.000 \mathrm{~km}^{2}$. O clima é úmido, pela proximidade com o oceano, com variações entre 19 e $28^{\circ} \mathrm{C}$ e precipitação anual média de $1.600 \mathrm{~mm},{ }^{26-28}$ o que explica as vazões específicas até cinco vezes maiores que do restante da bacia. Somente o Rio Gongogi, o principal afluente deste trecho, apresenta deflúvios que somam $40 \%$ dos totais anuais registrados na estação fluviométrica de Ubaitaba, que responde por $95 \%$ da área total da bacia do Rio de Contas $^{29}$ (Figura $1 \mathrm{~S}$, material suplementar).

Os principais usos das terras presentes, além de pequenos cultivos de subsistência, são o cultivo de cacau sombreado nos remanescentes florestais e a pecuária extensiva em áreas desfavoráveis a este. É uma região de baixos índices de desenvolvimento urbano e industrial e que abriga uma população de pouco menos de 130.000 habitantes, ${ }^{30}$ divididos em onze cidades de pequeno porte. Os efluentes eventualmente gerados pelas atividades antrópicas não sofrem, via de regra, nenhum tipo de tratamento prévio antes de atingirem os corpos hídricos receptores.

Interpretações utilizando diferentes bandas e composições espectrais de imagem Landsat de 2001, mostram que o estuário do Rio de Contas ocupa uma área muito pequena $\left(<2,0 \mathrm{~km}^{2}\right)$ comparada a toda a bacia, ou mesmo à bacia inferior. Nesta área o rio é controlado por um esporão, formado pelos sistemas de falhamentos que cortam as rochas granulíticas Arqueanas e os colocam em contato com terraços Quaternários da Formação Barreiras. ${ }^{23}$ Esta feição topográfica desaparece a menos de $1 \mathrm{~km}$ de sua foz. Considerando o baixo valor obtido na razão área da bacia/volume do estuário e também a reduzida penetração da cunha salina, mesmo em períodos de baixa vazão e maré de sizígia, ${ }^{31}$ o prisma de maré resultante é de pequena monta. Nestas circunstâncias, os processos biogeoquímicos que são típicos de regiões estuarinas como, por exemplo, denitrificação ou sedimentação de P particulado, têm reduzida oportunidade de operar modificações na carga continental, antes que essa seja exportada para águas costeiras adjacentes.

\section{Estimativas de cargas}

Fatores de emissão são as ferramentas preferencialmente utilizadas para a quantificação de cargas de contaminantes quando da inexistência de medidas sistemáticas in situ, sendo aplicáveis ao nível global, ${ }^{32-37}$ regional ${ }^{21,22,38-42}$ e local; $2,7,43,44$ sendo adotados como metodologia padrão por importantes agências ambientais. ${ }^{19,20,45,46}$ Nestas estimativas são levadas em consideração tanto fontes naturais quanto antrópicas. Para estimar emissões de fontes antrópicas são buscadas as relações entre as atividades produtivas e sua respectiva carga de elementos liberada no meio ambiente, tais relações são obtidas utilizando-se dados de produção e uso de insumos da agropecuária; ${ }^{13,47-52}$ volumes e concentrações disponíveis para efluentes e taxas de retenção em solos das bacias. ${ }^{7,22,53,54}$ As emissões associadas aos processos naturais edáficos e atmosféricos são estimadas levando-se em consideração a composição e distribuição de solos, taxas de liberação e/ou retenção das substâncias de interesse nos solos e equações de perda de solos..$^{3,755-60}$ No caso da contribuição atmosférica, quando da inexistência da medição in situ, são buscadas concentrações das substâncias de interesse na deposição atmosférica ou mesmo suas taxas de deposição em áreas com características ambientais semelhantes. Assim, de posse de taxas e do inventário de fontes naturais disponíveis na literatura, foram estimados os fatores de emissão, sempre que possível adotando valores locais ou regionais e recorrendo a valores globais apenas quando estes não estavam disponíveis para a área de estudo ou região. As respectivas equações e memória de cálculo para cada fonte e substância avaliada são detalhadas diretamente nas tabelas de resultados. Mais detalhes sobre o uso de fatores de emissão para estuários da região NE, suas limitações e aplicações podem ser encontrados na literatura especializada. ${ }^{21,22,41,42}$

\section{RESULTADOS E DISCUSSÃO}

As estimativas das cargas naturais dos nutrientes e metais estudados para as sub-bacias do rio Gongogi e Contas são apresentadas na Tabela 1. Duas são as fontes naturais dos elementos consideradas: a deposição atmosférica e a denudação física e química dos solos. Suas cargas estão diretamente relacionadas à área da bacia e à concentração elementar da deposição atmosférica total e à capacidade de retenção de elementos pelos solos locais, e pela composição química original dos diferentes tipos de solos presentes na bacia e suas áreas de cobertura, respectivamente. No caso dos solos ainda é levada em consideração a taxa de perda de solo. Os principais tipos de solos presentes na área estudada são podsólicos $\left(1.800 \mathrm{~km}^{2}\right)$, latossolos $\left(2.600 \mathrm{~km}^{2}\right)$ e solos brunos $\left(1.900 \mathrm{~km}^{2}\right){ }^{28}$ somando cerca de 6.300 $\mathrm{km}^{2}$. O restante da área incluindo solos alagados de mangues, praias e falésias não foram considerados neste estudo. Mangues são considerados áreas de acumulação de substâncias além de ocuparem uma área muito reduzida na bacia. Praias e falésias quando sofrem denudação exportam materiais diretamente para o mar. Solos tropicais planos sob agricultura não-mecanizada ou vegetação secundária apresentam perdas anuais médias ${ }^{61-63}$ da ordem de $130 \mathrm{t} \mathrm{km}^{-2}$. Entretanto, experimentos recentes realizados no sul da $\mathrm{Bahia}^{58}$ mediram taxas anuais variando de 48 a $123 \mathrm{t} \mathrm{km}^{-2}$. Devido à semelhança dos solos da Bacia do Rio de Contas, foi utilizada a média obtida daqueles experimentos de $75 \mathrm{t} \mathrm{km}^{-2}$ para o cálculo das estimativas de cargas por perda de solos. As concentrações médias dos elementos de interesse nas classes de solos presentes na bacia ${ }^{28}$ foram obtidas em diferentes estudos realizados no Brasil. ${ }^{11,12,58,64-71}$ 
Tabela 1. Contribuição das emissões naturais; deposição atmosférica ${ }^{i}$ e denudação física e química de solos ${ }^{\mathrm{ii}}$ de nutrientes e metais $\left(\mathrm{t}^{\mathrm{ano}} \mathrm{H}^{-1}\right)$ para as duas sub-bacias que compõem a bacia inferior do Rio de Contas

\begin{tabular}{|c|c|c|c|c|c|c|c|}
\hline \multicolumn{8}{|c|}{ GONGOGI } \\
\hline Fonte & $\mathrm{N}$ & $\mathrm{P}$ & $\mathrm{Zn}$ & $\mathrm{Cu}$ & $\mathrm{Pb}$ & $\mathrm{Cd}$ & $\mathrm{Hg}$ \\
\hline $\begin{array}{l}\text { Deposição } \\
\text { atmosférica }\end{array}$ & 139 & 9,7 & 26 & 4,0 & 5,3 & 7,3 & 0,03 \\
\hline Runoff de solos & 214 & 2,9 & 9,4 & 7,0 & 6,5 & 0,5 & 0,01 \\
\hline Total & 353 & 12,6 & 35,4 & 11,0 & 11,8 & 7,8 & 0,04 \\
\hline \multicolumn{8}{|c|}{ CONTAS } \\
\hline Fonte & $\mathrm{N}$ & $\mathrm{P}$ & $\mathrm{Zn}$ & $\mathrm{Cu}$ & $\mathrm{Pb}$ & $\mathrm{Cd}$ & $\mathrm{Hg}$ \\
\hline $\begin{array}{l}\text { Deposição } \\
\text { atmosférica }^{6}\end{array}$ & 71 & 5,0 & 14 & 2,0 & 2,7 & 3,8 & 0,01 \\
\hline Runoff de solos ${ }^{7}$ & 92 & 1,4 & 5,9 & 3,1 & 3,1 & 0,2 & 0,01 \\
\hline Total & 163 & 6,4 & 19,9 & 5,1 & 5,8 & 4,0 & 0,02 \\
\hline \multicolumn{8}{|c|}{ TOTAL PARA A BACIA INFERIOR } \\
\hline $\begin{array}{l}\text { Deposição } \\
\text { atmosférica }^{6}\end{array}$ & 210 & 14,7 & 40 & 6 & 8 & 11,1 & 0,04 \\
\hline Runoff de solos ${ }^{7}$ & 306 & 4,3 & 15,3 & 10,1 & 9,6 & 0,7 & 0,02 \\
\hline Total Natural & 516 & 19 & 55,3 & 16,1 & 17,6 & 11,8 & 0,06 \\
\hline
\end{tabular}

i - obtido pela média da deposição atmosférica relatada para áreas litorâneas de baixo ou nenhum desenvolvimento industrial ${ }^{3,55-57,59,71-76}$ ponderado pela pluviosidade anual real da bacia $(1.600 \mathrm{~mm})$ e corrigido por fatores de retenção de cada elemento no solo (N: 0,37; P: 0,30; Cu, Pb: 0,35; Zn: 0,45; Cd: 0,40; Hg: $0,90)^{37,47,73}$ e para a área das bacias..$^{27,28}$ ii - taxa de denudação média para solos planos tropicais litorâneos do sul da Bahia ${ }^{58}$ de $74 \mathrm{t} \mathrm{km}^{-2}$ ano $^{-1}$; concentração média de elementos por tipo de solo obtida pela média relatada para áreas litorâneas de baixo desenvolvimento industrial ${ }^{11,12,58,6470} \mathrm{e}$ análises realizadas durante o presente estudo ponderadas pela área de cobertura de cada tipos de solo. ${ }^{26}$

A deposição atmosférica foi calculada com base em uma precipitação médiaa ${ }^{26,27}$ de $1.600 \mathrm{~mm}^{2}$ ano $^{-1} \mathrm{e}$ a deposição total com base nas concentrações dos elementos típicas de áreas com baixa taxa de urbanização e industrialização, ${ }^{3,55-57,59,71-76}$ como é o caso do baixo curso do Rio de Contas. Os resultados foram corrigidos pela taxa de retenção de cada elemento pelos solos típicos da bacia. . $^{37,47,73}$

A derivação das contribuições destes dois aportes naturais mostra que a contribuição dos solos domina o aporte natural de $\mathrm{N}, \mathrm{Cu}$ e $\mathrm{Pb}$ e representa entre 10 a $80 \%$ do total para $\mathrm{Cd}, \mathrm{P}, \mathrm{Zn}$ e $\mathrm{Hg}$, para as duas sub-bacias estudadas (Tabela 1). Este resultado é devido às baixas concentrações destes últimos nos perfis pedológicos litorâneos do que um enriquecimento na precipitação. Resultados similares têm sido verificados em outras bacias costeiras do litoral NE do Brasil sob condições semelhantes de usos de solo, porém com contribuição relativamente menor dos solos que naquelas bacias que abrigam extensas áreas de agricultura mecanizada, como no Rio Jaguaribe (CE), por exemplo. ${ }^{21}$ Uma vez que os tipos de solos estão distribuídos de forma similar entre as das sub-bacias, as maiores cargas originadas na bacia do Rio Gongogi são resultantes da maior área desta bacia.

As cargas resultantes de atividades antrópicas estão apresentadas na Tabela 2. A atividade agrícola desponta como fonte majoritária para $\mathrm{N}, \mathrm{Pe} \mathrm{Cu}$, devido ao uso de diferentes insumos, particularmente fertilizantes para os dois primeiros e de defensivos agrícolas para o último, de forma preponderante na cultura cacaueira onde o $\mathrm{Cu}$ é ainda utilizado em larga escala como biocida. Por exemplo, perdas de $\mathrm{Cu}$ variando entre 15,3 a $516 \mathrm{~g} \mathrm{Cu} \mathrm{ha}^{-1} \mathrm{ano}^{-1}$ foram relatadas, para plantações de cacau na região de Ilhéus. ${ }^{8}$ As cargas anuais estima- das destes três elementos originadas na agricultura foram de 1.119, 1.218 e $9 \mathrm{t} \mathrm{ha}^{-1}$, respectivamente. A pecuária e a disposição de águas servidas são duas outras fontes com participação significativa nos totais liberados devido à intervenção humana, com destaque para o $\mathrm{N}$ oriundo da liberação de águas servidas $\left(447\right.$ t ha $^{-1}$ ano $\left.^{-1}\right)$ e P e Zn (208 e 7,1 t ha ${ }^{-1}$, respectivamente) pela criação de gado, principalmente bovino, atividade dominante na bacia do Rio Gongogi. A disposição inadequada de resíduos sólidos urbanos aparece como fonte principal para $\mathrm{Pb}, \mathrm{Cd}$ e $\mathrm{Hg}$, embora este último apresente carga semelhante originada na disposição de águas servidas. As menores cargas foram estimadas para o escoamento superficial urbano, resultante do processo de lavagem das superfícies urbanas, e que é um reflexo do baixo índice de urbanização dos municípios da região.

Tabela 2. Contribuição das emissões antrópicas das diferentes fontes de nutrientes e metais $\left(\mathrm{t} \mathrm{ano}^{-1}\right)$ para as duas sub-bacias que compõem a bacia inferior do Rio de Contas

\begin{tabular}{|c|c|c|c|c|c|c|c|}
\hline \multicolumn{8}{|c|}{ GONGOGI } \\
\hline Fonte & $\mathrm{N}$ & $\mathrm{P}$ & $\mathrm{Zn}$ & $\mathrm{Cu}$ & $\mathrm{Pb}$ & $\mathrm{Cd}$ & $\mathrm{Hg}$ \\
\hline Agricultura $^{1}$ & 453 & 380 & 0,11 & 3,5 & 0,01 & 0,04 & $<0,001$ \\
\hline Águas servidas² & 269 & 77 & 1,5 & 0,7 & 0,29 & 0,06 & 0,018 \\
\hline Pecuária ${ }^{3}$ & 128 & 185 & 6,2 & 1,0 & 0,12 & 0,01 & $<0,001$ \\
\hline $\begin{array}{l}\text { Resíduos } \\
\text { sólidos }{ }^{4}\end{array}$ & 27 & 68 & 3,6 & 1,1 & 2,3 & 0,23 & 0,018 \\
\hline Runoff urbano ${ }^{5}$ & 4,6 & 0,7 & 0,2 & 0,01 & 0,02 & $<0,01$ & $1<0,001$ \\
\hline $\begin{array}{l}\text { Total para a } \\
\text { sub-bacia }\end{array}$ & 882 & 711 & 12 & 6,3 & 2,7 & 0,34 & 0,036 \\
\hline \multicolumn{8}{|c|}{ CONTAS } \\
\hline Fonte & $\mathrm{N}$ & $\mathrm{P}$ & $\mathrm{Zn}$ & $\mathrm{Cu}$ & $\mathrm{Pb}$ & $\mathrm{Cd}$ & $\mathrm{Hg}$ \\
\hline Agricultura $^{1}$ & 666 & 838 & 0,1 & 5,5 & 0,001 & 0,01 & $<0,001$ \\
\hline Águas servidas ${ }^{2}$ & 178 & 51 & 1,1 & 0,5 & 0,19 & 0,042 & 0,012 \\
\hline Pecuária ${ }^{3}$ & 32 & 23 & 0,9 & 0,2 & 0,021 & 0,001 & $<0,001$ \\
\hline $\begin{array}{l}\text { Resíduos } \\
\text { sólidos }\end{array}$ & 22 & 45 & 1,8 & 0,7 & 1,51 & 0,015 & 0,012 \\
\hline Runoff urbano ${ }^{5}$ & 3,5 & 0,5 & 0,1 & 0,01 & 0,018 & $<0,001$ & $1<0,001$ \\
\hline $\begin{array}{l}\text { Total para a } \\
\text { sub-bacia }\end{array}$ & 901 & 957 & 4,0 & 6,9 & 1,7 & 0,07 & 0,024 \\
\hline \multicolumn{8}{|c|}{ TOTAL PARA A BACIA INFERIOR } \\
\hline Total Antrópico & 1.783 & 1.669 & 16,0 & 13,2 & 4,4 & 0,41 & 0,06 \\
\hline
\end{tabular}

${ }^{1}$ Aplicação como insumo ou impureza; ${ }^{48-50,79,80}$ área por tipo de cultivar. ${ }^{30}$ Fatores de retenção no solo..$^{37,47,73}{ }^{2}$ Concentração em águas servidas; ${ }^{6,54,81,82}$ consumo/emissão de água servida; ${ }^{83}$ número de habitantes. ${ }^{30}{ }^{3}$ Tipo e tamanho do rebanho, ${ }^{30}$ produção de dejetos por animal ${ }^{84}\left(\mathrm{~kg} \mathrm{cabeça}^{-1} \mathrm{dia}^{-1}\right)$; concentração dos elementos em dejetos. ${ }^{13,85}{ }^{4}$ Concentração dos elementos em rejeitos sólidos urbanos; ${ }^{33,86}$ produção de rejeitos por habitante; ${ }^{1,3,53}$ número de habitantes. ${ }^{30}$ Pluviosidade anual; ${ }^{28}$ concentração de elementos no runoff urbano. ${ }^{5-7}$

Uma comparação entre as duas sub-bacias, mostra a do Rio Gongogi, que representa $66 \%$ da área total da bacia inferior e contribui com o principal deflúvio anual registrado, ${ }^{27,28}$ como a principal contribuidora das cargas antrópicas dos elementos estudados para a bacia inferior do Rio de Contas. Apenas a agricultura apresenta contribuição maior na sub-bacia do Rio de Contas. A menor aptidão para cultivos agrícolas, na sub-bacia do Rio Gongogi, faz com que esta vasta área seja ocupada principalmente por pastagens, resultando 
na pecuária como principal fonte de todos os elementos estudados. Da mesma forma, por apresentar um maior número de habitantes e mais áreas urbanizadas, esta sub-bacia também apresenta cargas bem maiores de todos os elementos das fontes relacionadas à urbanização ( $\mathrm{Pb}, \mathrm{Cd} \mathrm{e} \mathrm{Hg})$.

A comparação entre as cargas naturais e antrópicas dos elementos estudados para a bacia inferior do Rio de Contas (Tabela 3) revela um predomínio das atividades antrópicas nos lançamentos de nutrientes, com contribuição de 99 e $78 \%$ para $\mathrm{P}$ e N, respectivamente. No caso específico do P, com ínfima fração de origem natural, o uso de fertilizantes na agricultura, mesmo levando em consideração o baixo nível de mecanização dos cultivos locais, representa até $72 \%$ da carga total lançada nos corpos hídricos da área estudada, sendo duas ordens de grandeza maior que a carga originada da lixiviação natural dos solos, por exemplo. Com exceção do runoff urbano que representa pequena fração da emissão total do $\mathrm{P}$, as demais fontes antrópicas consideradas, embora menores que a atividade agrícola, também são significativas quando comparadas ao aporte natural, com cargas variando entre cinco e dez vezes os aportes naturais.

Tabela 3. Comparação entre as cargas totais $\left(\mathrm{t}\right.$ ano $\left.^{-1}\right)$ de metais e nutrientes de origem natural e antrópica para a bacia inferior do Rio de Contas (BA)

\begin{tabular}{lccccccc}
\hline Fonte & $\mathrm{N}$ & $\mathrm{P}$ & $\mathrm{Zn}$ & $\mathrm{Cu}$ & $\mathrm{Pb}$ & $\mathrm{Cd}$ & $\mathrm{Hg}$ \\
Total Natural & 516 & 19 & 55,3 & 16,1 & 17,6 & 11,8 & 0,06 \\
Total Antrópico & 1.783 & 1.669 & 16,0 & 13,2 & 4,4 & 0,41 & 0,06 \\
$\begin{array}{l}\text { Total para a } \\
\text { bacia inferior }\end{array}$ & 2.299 & 1.688 & 71,3 & 29,3 & 22,0 & 12,2 & 0,12 \\
$\begin{array}{l}\text { \% Antrópico } \\
\text { no total }\end{array}$ & 78 & 99 & 23 & 45 & 20 & 3 & 50 \\
\hline
\end{tabular}

Para N o quadro é semelhante, quanto ao domínio da origem antrópica e da maior participação da atividade agrícola (49\%). Embora outras atividades antrópicas, particularmente a disposição de águas servidas (19\%) também contribuam com cargas significativas e bem maiores que o aporte natural deste elemento.

As estimativas apresentadas situam a atividade agrícola, secundada pela pecuária, como principais fontes de nutrientes para a bacia inferior e estuário do Rio de Contas e, portanto, agentes potenciais para a eutrofização desse corpo hídrico. Estas elevadas cargas já promovem um desequilíbrio na estrutura dos ecossistemas e a existência de extensos bancos de macrófitas na região do baixo Rio de Contas, já é um claro indicador deste processo. Esses aportes devem igualmente deixar uma forte assinatura na hidroquímica fluvial e estuarina. Dados ainda não publicados sobre a concentração de nutrientes dissolvidos nas águas da região estuarina do Rio de Contas indicam que as estimativas de cargas realizadas são coerentes com as concentrações medidas no campo. Concentrações variando entre 3,1 e 5,2 $\mu \mathrm{M}$ de $\mathrm{N}$ total e entre 0,2 e $0,4 \mu \mathrm{M}$ de $\mathrm{P}-\mathrm{PO}_{4}$ foram encontradas nos pontos situados na porção final da bacia e que associados a valores de até 5,3 $\mu \mathrm{g} \mathrm{L}^{-1}$ de Clorofila-a, já indicam um ambiente com características de mesotróficas a eutróficas, segundo índices preconizados na literatura e por agências ambientais. ${ }^{46,77}$ Fluxos instantâneos positivos para o oceano de $\mathrm{N}$ e $\mathrm{P}$ de até 45 e $25 \mathrm{~kg} \mathrm{ha}^{-1}$, respectivamente, também foram estimados para a bacia inferior do Rio de Contas. ${ }^{31}$

Dentre os metais estudados associados a fontes não oriundas da agropecuária, nenhum apresenta cargas antrópicas superiores àquelas de origem natural, com exceção do Hg que apresenta cargas equivalentes das duas origens. Neste caso, a disposição inadequada de resíduos sólidos urbanos é a principal responsável pelos aportes antrópicos (86, 80 e $50 \%$ da carga antrópica total para $\mathrm{Pb}, \mathrm{Cd}$ e $\mathrm{Hg}$, respectivamente).
A sub-bacia do Rio Gongogi, por apresentar uma maior densidade de núcleos urbanos é a principal origem das cargas destes metais.

A Tabela 4 apresenta as cargas estimadas, normalizadas pela área total da bacia inferior do Rio de Contas, sendo que esta normalização permite a comparação direta com outras bacias estudadas no NE do Brasil. Quando comparado com estudos semelhantes, conduzidos em outros estados do NE brasileiro, ${ }^{21,22}$ apesar de algumas diferenças pontuais ligadas ao número de habitantes por bacias, tipos de fontes presentes, índices pluviométricos ou tipos de solos predominantes, a primeira constatação é a de que, igualmente no Rio de Contas, o aporte de nutrientes de origem antrópica já supera aqueles de origem natural. Esta constatação foi verificada para nutrientes (N e P) em 16 outras bacias do NE brasileiro. ${ }^{21,22}$ As cargas totais de origem antrópica em todas as bacias estudadas no NE do Brasil, com exceção das grandes regiões metropolitanas das capitais de Estado, são dominadas por emissões agrícolas, seguido pela pecuária, da mesma forma que para a bacia inferior do Rio de Contas. Nas bacias metropolitanas, entretanto, dominam as cargas originadas na disposição de águas servidas. Para os metais, novamente com exceção das regiões metropolitanas, as fontes naturais são as principais responsáveis pelas cargas estimadas. No caso do $\mathrm{Pb}, \mathrm{Cd}$ e $\mathrm{Hg}$, as emissões antrópicas são, via de regra, dominadas pelas emissões associadas à disposição inadequada de resíduos sólidos urbanos.

Tabela 4. Fatores de emissão, normalizados por unidade de área ( $\mathrm{kg}$ $\mathrm{km}^{-2} \mathrm{ano}^{-1}$ ) de nutrientes e metais originados de processos naturais e antrópicos na bacia inferior do Rio de Contas, BA. Valores arredondados à unidade para $\mathrm{N}$ e $\mathrm{P}$

\begin{tabular}{|c|c|c|c|c|c|c|c|}
\hline Fonte & $\mathrm{N}$ & $\mathrm{P}$ & $\mathrm{Zn}$ & $\mathrm{Cu}$ & $\mathrm{Pb}$ & $\mathrm{Cd}$ & $\mathrm{Hg}$ \\
\hline $\begin{array}{l}\text { Lixiviação } \\
\text { dos solos }\end{array}$ & 43 & 1 & 2,2 & 1,4 & 1,4 & 0,1 & 0,003 \\
\hline $\begin{array}{l}\text { Deposição } \\
\text { atmosférica }\end{array}$ & 30 & 2 & 5,6 & 0,8 & 1,1 & 1,6 & 0,006 \\
\hline Total natural & 73 & 3 & 7,8 & 2,2 & 2,5 & 1,7 & 0,009 \\
\hline Agricultura & 158 & 172 & 0,1 & 1,3 & 0,00 & 0,01 & 0,000 \\
\hline Pecuária & 63 & 18 & 0,4 & 0,2 & 0,07 & 0,01 & 0,004 \\
\hline Águas servidas & 23 & 29 & 1,0 & 0,2 & 0,02 & 0,00 & 0,000 \\
\hline $\begin{array}{l}\text { Resíduos } \\
\text { sólidos urbanos }\end{array}$ & 7 & 16 & 0,8 & 0,3 & 0,54 & 0,03 & 0,004 \\
\hline Runoff urbano & 1 & 0 & 0,0 & 0,0 & 0,01 & 0,00 & 0,000 \\
\hline Total antrópico & 252 & 235 & 2,3 & 2,0 & 0,6 & 0,05 & 0,008 \\
\hline TOTAL & 325 & 238 & 10,1 & 4,2 & 3,1 & 1,8 & 0,017 \\
\hline
\end{tabular}

No caso dos metais com forte contribuição agrícola (Cu e Zn), as cargas estimadas para o Rio de Contas coincidem com a faixa mais baixa de emissões calculadas para as bacias de outros Estados do NE com forte desenvolvimento de agricultura irrigada e mecanizada, como no Rio Jaguaribe (CE), particularmente a fruticultura irrigada. $^{21,22}$

De maneira geral, observa-se para a região do baixo Rio de Contas que os aportes de alguns metais ( $\mathrm{Cu}$ e $\mathrm{Zn}$, principalmente) oriundos de diferentes usos não urbanos da terra já igualam ou são muito próximos às cargas resultantes apenas de processos naturais. Dados preliminares de um estudo em andamento sobre a distribuição de metais em sedimentos no trecho estuarino da bacia do Rio de Contas corroboram estes resultados e indicam um incremento significativo de concentrações de metais quando comparado aos valores medidos a montante da represa da UHE de Funil e mesmo no primeiro trecho do rio logo abaixo da represa. Em direção à foz 
do rio, as concentrações de $\mathrm{Zn}$ aumentam de 4 para 10 e até $34 \mu \mathrm{g}$ $\mathrm{g}^{-1}$, enquanto as de $\mathrm{Cu}$ de 3 para $10 \mu \mathrm{g} \mathrm{g}^{-1}$. Também tem sido ressaltado o assoreamento do baixo curso do Rio de Contas, ${ }^{78}$ o que está de acordo com as elevadas contribuições naturais originadas na denudação física e química dos solos da bacia. Estas evidências, de forma similar àquelas obtidas para $\mathrm{N}$ e $\mathrm{P}$, ressaltam a capacidade de alteração do ambiente biogeoquímico da bacia inferior do Rio de Contas pela atividade antrópica.

\section{CONCLUSÕES}

Os valores obtidos no presente trabalho devem ser considerados como uma primeira aproximação nos cálculos das cargas de elementos para a porção inferior e estuarina e região costeira associada do Rio de Contas. Deverão ser, entretanto, atualizados pela adoção de fatores de emissão melhor ajustados à realidade regional e seu desenvolvimento e pela determinação no local dos dados referentes à deposição atmosférica e concentração de elementos nos diferentes tipos de solos presentes na bacia. Para sua validação final, são necessários estudos sistemáticos sobre a biogeoquímica das águas e sedimentos dos corpos hídricos existentes. Embora não tenham sido considerados os aportes oriundos da bacia como um todo, uma estimativa desta contribuição também irá melhorar a exatidão destas estimativas. Entretanto, todos estes ajustes não irão modificar o panorama geral, principalmente no que tange à detecção de emissões antrópicas maiores que as de origem natural, mesmo para uma região que apresenta baixos índices de desenvolvimento econômico e social.

Os lançamentos calculados para a maioria dos metais não apresentam magnitudes comparáveis àquelas presentes em regiões industrializadas normalmente associadas a esses lançamentos. Mesmo assim o uso de insumos na agropecuária resulta em cargas de Zn e $\mathrm{Cu}$ já equivalentes aos aportes naturais, enquanto que no caso do $\mathrm{Hg}$ oriundo de fontes urbanas, as cargas antrópicas já são iguais à contribuição de fontes naturais.

Ao contrário dos metais, os aportes de nutrientes ( $\mathrm{Ne} \mathrm{P}$ ) oriundos dos usos da terra no baixo Rio de Contas, da mesma forma que em outras bacias do NE brasileiro, já superam em muito os aportes naturais, despontando a agricultura e os esgotos domésticos como principal fonte de $\mathrm{N}$ e a agropecuária como principal fonte de $\mathrm{P}$. Estes aportes já são responsáveis pelo processo de eutrofização já evidenciado na bacia inferior. Finalmente, a sub-bacia do Rio Gongogi, devido à sua maior área e também aos usos da terra presentes em sua área de captação, é a principal responsável pelos aportes calculados e, assim, se configura em área chave a ser considerada em eventuais planos de manejo e implementação de políticas públicas de gestão ambiental.

Devido às características geomorfológicas da região estuarina, os materiais de origem continental não sofrem maiores transformações antes de atingirem a região costeira adjacente à foz do Rio de Contas. A reduzida área ocupada por seu estuário e seu prisma de maré de pequena amplitude, o que limita a ação da dinâmica biogeoquímica que caracteriza este ecótono, resulta em uma exportação líquida destes materiais para a plataforma continental adjacente.

\section{MATERIAL SUPLEMENTAR}

A localização da bacia hidrográfica inferior do Rio de Contas, composta pelas sub-bacias dos Rios Gongogi e de Contas, sua posição entre as regiões hidrográficas da Bahia, distribuição e área dos municípios componentes e localização de suas sedes está disponível gratuitamente em http://quimicanova.sbq.org.br, na forma de arquivo .PDF, com acesso livre.

\section{AGRADECIMENTOS}

Este estudo faz parte dos resultados obtidos no âmbito do Projeto Instituto do Milênio; Transferência de Materiais na Interface Continente-Oceano, Proc. No. 420.050/2005-1, e dos objetivos do Instituto Nacional de Ciência e Tecnologia de Transferência de Materiais Continente-Oceano, Proc. No. 573.601/2008-9, financiado pelo CNPq. Os autores agradecem ao Editor Associado de Química Nova e a dois assessores anônimos que muito contribuíram para melhorar uma versão inicial deste manuscrito.

\section{REFERÊNCIAS}

1. Machado, W.; Moscatelli, M.; Rezende, L. G.; Lacerda L. D.; Environ. Pollut. 2002, 120, 455.

2. Oygard, J. K.; Mage, A.; Gjengedal, E.; Water Res. 2004, 38, 2851.

3. Silva Filho, E. V.; Sella, S. M.; Spinola, E. C.; Santos, I. R.; Machado, W.; Lacerda, L. D.; Microchem. J. 2006, 82, 196.

4. Hutton, M.; Symon, C. ; Sci. Total Environ. 1986, 57, 129.

5. Davis, A. P.; Shokouhian, M.; Shubei, M.; Chemosphere 2001, 44, 997.

6. Sorme, L.; Lagerkvist, R.; Sci. Total Environ. 2002, 298, 131.

7. Prestes, E. C.; Anjos, V. E.; Sodré, F. F.; Grassi, M. T.; J. Braz. Chem. Soc. 2006, 17, 53.

8. Lima, J. S.; Lichtig, J.; Oliveria, E.; Menk, J.R.F.; Rev. Ceres. 1999, 46, 571.

9. Gimeno-García, E.; Andreu, V.; Boluda, R.; Environ. Pollut. 1996, 92, 19.

10. Mortvedt, J. J.; Fertilizer Res. 1996, 43, 55.

11. Ramalho, J. F. G. P.; Sobrinho, N. M. B. A.; Floresta \& Ambiente 2001, 8,120 .

12. Ramalho, J. F. G. P.; Sobrinho, N. M. B. A.; Velloso, A. C. X.; Pesq. Agropec. Brasil. 2000, 35, 1289.

13. Menzi, H.; Kessler, J.; Proceedings of the $8^{\text {th }}$ International Conference of the FAO Network on Recycling of Agricultural. Municipal and Industrial Residues in Agriculture, Rome, Itália, 1998.

14. Nicholson, F. A.; Chambers, B. J.; Williams, J. R.; Unwin, R. J.; Bioresour. Technol. 1999, 70, 23.

15. Paiva, D. P.; Anais do Seminário Internacional de Aves e Suínos, Florianópolis, Brasil, 2005

16. Lacerda, L. D.; Biogeochemistry of Trace Metal and Diffuse Pollution in Mangrove Ecosystems, International Society for Mangrove Ecosystems: Okinawa, 1998.

17. Brasil, Divisão Hidrográfica Nacional; Resolução $n^{\circ} 32$ (15/10/03), Conselho Nacional de Recursos Hídricos: Brasília, 2003.

18. LOICZ; Land-Ocean Interaction in the Coastal Zone: Science Plan and Implementation Strategy. IGBP Report 51 / IHDP Report 18: Stockholm, 2005.

19. NRC; Clean Coastal Waters. Understanding and Reducing the Effects of Nutrient Pollution. National Research Council, Academy Press: Washington, 2003.

20. EEA; Nutrients in European Ecosystems. Environmental Assessment Report No. 4. European Environmental Agency, Office for Official Publications of the European Communities: Luxemburg, 1999.

21. Lacerda, L. D.; Molisani, M. M.; Sena, D.; Maia, L. P.; Environ. Monitor. Asses. 2008, 141, 149.

22. Lacerda, L. D.; Braz. J. Aquat. Sci. Technol. 2006, 10, 13.

23. Barbosa, J. S. F.; Dominguez, J. M. L.; Geologia da Bahia. Texto Explicativo, Superintendência de Geologia e Recursos Minerais: Salvador, 1996.

24. CRA; Qualidade das Águas da Bacia Hidrográfica do Rio de Contas, Centro de Recursos Ambientais do Estado da Bahia: Salvador, 2000.

25. CRA; Qualidade das Águas da Bacia Hidrográfica do Rio de Contas, Centro de Recursos Ambientais do Estado da Bahia: Salvador, 2001.

26. SEI; Atributos Climáticos do Estado da Bahia, Superintendência de Estudos Econômicos e Sociais da Bahia: Salvador, 1988. 
27. SEI; Bacias Hidrográficas do Estado da Bahia, Superintendência de Estudos Econômicos e Sociais da Bahia: Salvador, 2006a.

28. SEI; Recursos Minerais do Estado da Bahia, Superintendência de Estudos Econômicos e Sociais da Bahia: Salvador, 2006b.

29. Paula, F. C. F.; Figueiredo, A. F.; Anais do XII Congresso Latino Americano de Ciências do Mar, Florianópolis, Brasil, 2007.

30. http://www.ibge.gov.br/cidadesat/topwindow.htm?1, acessada em Outubro 2008.

31. Paula, F. C. F.; Ovalle, A. R. C.; Bernardes, M. C.; Medeiros, P. R.; Pedrosa, P.; Souza, W. F. L.; Lacerda, L. D.; Anais do XII Congresso Latino Americano de Ciências do Mar, Florianópolis, Brasil, 2007.

32. Nriagu, J. O.; Nature 1989, 338, 47.

33. Nriagu, J. O.; Pacyna, J. M.; Nature 1988, 333, 134.

34. Bouwman, A. F.; Lee, D. S.; Asman, A. H.; Dentener, F. J.; van der Hoek, K. W. A.; Olivier, J. G. J.; Global Biogeochem. Cycl. 1997, 11, 561.

35. Pirrone, N.; Allegrini, I.; Keeler, G. J.; Nriagu, J. O.; Rossmann, R.; Robbins, J. A. ; Atmos. Environ. 1998, 32, 929.

36. Pirrone, N.; Keeler, G. J.; Nriagu, J. O.; Atmos. Environ. 1996, 30, 2981.

37. Lacerda, L. D.; Environ. Geol. 2003, 43, 308.

38. Lacerda, L. D.; Marins, R. V. ; J. Geochem. Explor. 1997, 58, 223.

39. Bricker, S. B.; Clement, C. G.; Pirhalla, D. E.; Orlansdo, S. P.; Farrow, D. R. G.; National Estuarine Eutrophication Assessment: effects of Nutrient Enrichment in the Nation's Estuaries, NOAA: Silver Spring, 1999.

40. Vaisman, A. G.; Lacerda, L. D.; Region. Environ. Change. 2003, 3, 140.

41. Lacerda, L. D.; Santos, J. A.; Madrid, R. M.; Mar. Pollut. Bull. 2006, $52,1823$.

42. Lacerda, L. D.; Santos, A. F.; Marins, R. V.; Quim. Nova 2007, 30, 366. 43. Marins, R. V.; Lacerda, L. D.; Villas Boas, R. C.; Ciênc. Cult. 1998, 50, 293.

44. Marins, R. V.; Lacerda, L. D.; Villas Boas, R. C. Em Mercury Contaminated Sites: Characterization. Risk Assessment and Remediation; Ebinhaus, R.; Turner, R. R.; Lacerda, L. D.; Vasiliev, O.; Salomons, W., eds.; Springer Verlag: Berlin, 1999, p. 207.

45. CETESB; Relatório de Atividades da Divisão de Fatores e Padrões de Emissão, Companhia Estadual de Tecnologia e Saneamento Básico: São Paulo, 1986.

46. EPA; National Recommended Water Quality Criteria, EPA822-R-02-047, Environmental Protection Agency, Office of Water: Washington, 2002.

47. Malavolta, E.; Dantas, J. P. Em Melhoramento e Produção do Milho no Brasil; Paterniani, E., ed.; Fundação Cargill: São Paulo, 1980, cap. 13.

48. Soto-Jimenez, M.; Paez-Osuna, F.; Ruiz-Fernandez, A. C.; Environ. Pollut. 2003, 125, 423.

49. Ferreira. J. M. S.; Produção Integrada de Coco, EMBRAPA Tabuleiros Costeiros: Aracaju, 2003.

50. Silva, E. C.; Silva Filho, A. V.; Almeida, M. A. R.; Ciênc. Agrotecnol. 2000, 24, 509.

51. Lima, J. S. L.; Agricul. Ecosyst. Environ. 1994, 48, 19.

52. Tundisi, J. G. ; Água no Século XXI: Enfrentando a Escassez, Rima: São Carlos, 2006.

53. ABES; Catálogo Brasileiro de Engenharia Sanitária, Associação Brasileira de Engenharia Sanitária e Ambiental: Rio de Janeiro, 1983.

54. von Sperling, M.; Introdução à Qualidade das Águas e ao Tratamento de Esgotos, Universidade Federal de Minas Gerais: Belo Horizonte, 1996.

55. Mello, W. Z.; Environ. Pollut. 2001, 114, 235.

56. Mello, W. Z.; Almeida, M. D.; Environ. Pollut. 2004, 129, 63.

57. Marins R. V.; Silva Filho, E. V.; Lacerda, L. D.; J. Braz. Chem. Soc. 1996, 7, 177.
58. Inácio, E. S. B.; Cantalice, J. R. B.; Nacif, P. G. S.; Araújo, Q. R.; Barreto, A. C.; R. Bras. Eng. Agríc. Ambiental 2007, 11, 355.

59. Tan, P. A.; Wong, A. Y. S.; Water, Air, Soil Pollut. 2000, 122, 261.

60. Magliavacca, D. M.; Teixeira, E. C.; Machado, A. C. M.; Pires, M. R.; Quim. Nova 2005, 28, 371.

61. Greenland, D. J.; Hall, R.; Soil Conservation and Management in the Humid Tropics, Willey: Chischester, 1977.

62. Goudie, A.; The Human Impact: on the Natural Environment, MIT Press: Cambridge, 1987.

63. Souza, W. P.; Dissertação de Mestrado, Universidade Estadual do Norte Fluminense, Brasil, 2000.

64. Dias, C. M. F.; Souza, C. M. M.; Moneratti, P. H.; Anais do X Congresso Brasileiro de Geoquímica, Curitiba, Brasil, 2001.

65. Holmgren, G. G. S.; Meyer, M. W.; Chaney, R. L.; Daniels, R. B.; J. Environ. Qual. 1993, 22, 335.

66. Silva, L. F.; Solos Tropicais: Aspectos Pedológicos, Ecológicos e de Manejo, Terra Brasilis: São Paulo, 1996.

67. Canellas, L. P.; Velloso, A. C. X.; Marciano, C. R.; Ramalho, J. F. G. P.; Rumjanek, V. M.; Rezende, C. E.; Santos, G. A.; R. Bras. Ci. Solo. 2003, 27, 935.

68. Mesquita, E. E.; Pinto, J. C.; Neto, A. E. F.; Santos, I. P. A.; Tavares, V. B.; R. Bras. Zootec. 2004, 33, 290.

69. Andrade, C. A.; Mattiazzo, M. E.; Scien. Florest. 2000, 58, 59.

70. Fadigas, F. S.; Sobrinho, N. M. B. A.; Mazur, N.; Anjos, L. H. C.; Freixo, A. A.; Rev. Bras. Eng. Agríc. Ambiental 2006, 10, 699.

71. Brunner, U.; Technol. Environ. Chem. 1998, 167, 171.

72. Burns, D. A.; Environ. Pollut. 2004, 127, 257.

73. Golley, F. B.; Mc Guiness, J. T.; Clements, R. G.; Child, G.I.; Duever, M. J.; Ciclagem de Minerais em um Ecossistema de Floresta Tropical Úmida, EDUSP: São Paulo, 1978.

74. Johnson, D. W.; Lindberg, S. E.; Atmospheric Deposition and Forest Nutrient Cycling, Springer Verlag: Berlin, 1998.

75. Lindberg, S.; Harris, R. C.; Water, Air, Soil Pollut. 1981, 16, 13.

76. Schlesinger, W. H.; Gray, J. T.; Gilliam, F. S.; Wat. Resour. Res. 1982 , $18,623$.

77. Tundisi, J. G.; Tundisi, T. M.; Limnologia, Oficina de Textos: São Paulo, 2008.

78. Campos, E. M.; Dissertação de Mestrado, Universidade Federal da Bahia, Brasil, 2001.

79. SEAGRI; Cultura da Manga, Secretaria de Agricultura Irrigação e Reforma Agrária do Estado da Bahia: Salvador, 2003.

80. McBride, M. B.; Spiers, G.; Comm. Soil Sci. Plant Anal. 2001, 32, 139.

81. Vollenweider, R. A.; Scientific Fundamentals of the Eutrophication of Lakes and Flowing Waters with Particular Reference to Nitrogen and Phosphorus as Factors in Eutrophication, OECD: Paris, 1968.

82. I. C. Consultants Ltd.; Pollutants in Urban Waste Water and Sewage Sludge, The Office for Official Publications of the European Communities: Luxembourg, 2001.

83. Doll, P.; Hauschild, M.; Reg. Environ. Change. 2002, 2, 150

84. Boyd, C. E.; Amer. Fish. Soc. Spec. Publ. 1971, 8, 153.

85. Ambrus, A.; Hamilton, D. J.; Kuiper, H. A.; Racke, K. D.; Pure Appl. Chem. 2003, 75, 937.

86. Binner, E.; Lechner, P.; Ziegler, C.; Riehl-Herwirsch, G.; Breitenau Landfill - Water Balance, Emissions and a Look into the Landfill Body, Breitenau Landfill Co.: Viena, 1996. 


\section{EMISSÕES NATURAIS E ANTRÓPICAS DE METAIS E NUTRIENTES PARA A BACIA INFERIOR DO RIO DE}

\section{CONTAS, BAHIA}

Francisco C. F. de Paula

Departamento de Ciências Agrárias e Ambientais, Universidade Estadual de Santa Cruz, 45650-000 Ilhéus - BA, Brasil

Luiz Drude de Lacerda*, Rozane V. Marins e José E. Aguiar

Instituto de Ciências do Mar, Universidade Federal do Ceará, 60165-081 Fortaleza - CE, Brasil

Álvaro R. C. Ovalle

Centro de Ciências Biológicas e Biotecnologia, Universidade Estadual do Norte Fluminense, 28015-620 Campos dos Goytacazes

- RJ, Brasil

Cézar A. T. Falcão Filho

Universidade Estadual de Santa Cruz, 45650-000 Ilhéus - BA, Brasil

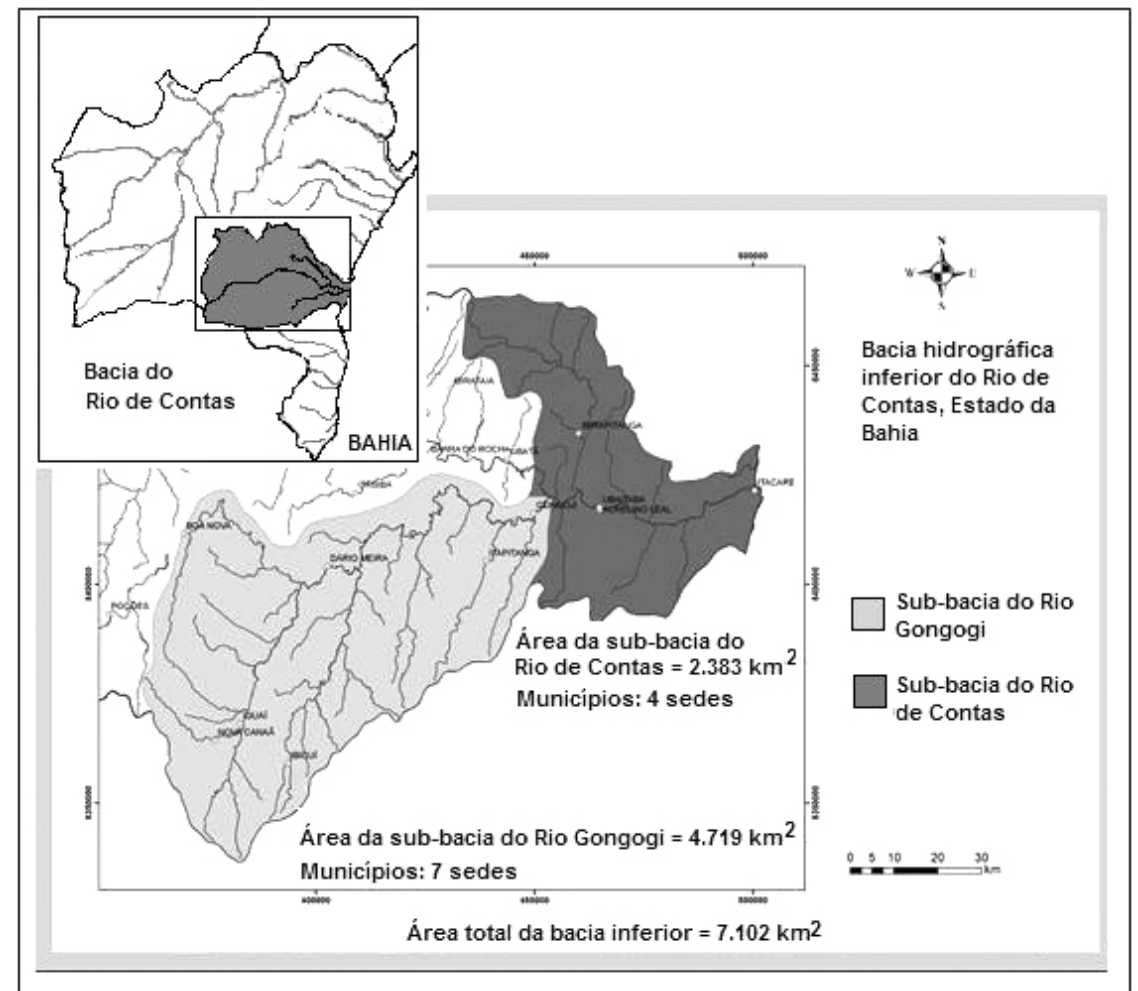

Figura 1S. Localização da bacia hidrográfica inferior do Rio de Contas, composta pelas sub-bacias dos Rios Gongogi e de Contas e sua posição entre as regiões hidrográficas da Bahia. Adaptado de original disponível em: www.sei.ba.gov.br./cartogramas ${ }^{27}$ 